\title{
Thick (3D) Sample Imaging Using iDPC-STEM at Atomic Scale
}

\author{
Ivan Lazić ${ }^{1}$, Eric G.T. Bosch ${ }^{1}$, Emrah Yücelen ${ }^{1}$, Robert Imlau ${ }^{1}$ and Lazar Sorin ${ }^{1}$
}

1. Thermo Fisher Scientific, Materials \& Structural Analysis, Eindhoven, The Netherlands.

STEM imaging techniques including (HA)ADF- and iDPC-STEM are well understood and applicable for thin specimen $[1,2,3]$. Both techniques can be used simultaneously, where the iDPC image shows both light and heavy atoms together in a thin sample at sub- $\AA$ resolution [4] and (HA)ADF-STEM emphasizes the heavier atoms.

For samples thicker than several tens of nanometers, the interpretation of STEM images, including (HA)ADF and iDPC STEM becomes more demanding. In order to cope with and resolve this challenge we introduce Through Focus Series (TFS) iDPC-STEM imaging. The TFS iDPC-STEM can be acquired simultaneously with TFS ADF-STEM [5, 6]. The TFS is obtained by placing the crossover of the probe at various depths of the sample by setting different probe defocus values. This depth sectioning is also common in confocal STEM [7]. Our investigations show that applying this TFS approach to iDPCSTEM enables clear interpretation of the obtained data, whereas the 3D information from ADF is very limited. Therefore, including iDPC extends our ability to image thick samples (up to $100 \mathrm{~nm}$ ) as well as to obtain their full 3D volume.

Here, we present how TFS iDPC-STEM reveals the three dimensional atomic structure of thick crystalline samples. We reveal defects and crystal variations at different depths of the various materials. The TFS iDPC-STEM results are compared to simultaneous and complementary TFS ADF-STEM as well as related to conventional confocal STEM [7]. Resolution in z direction as well as theoretical interpretation based on exact thin sample STEM theory [3] will be discussed.

Figure 1 shows an example of TFS iDPC-STEM imaging of a $25 \mathrm{~nm}$ thick SrTiO3 [011] sample. In this case focus steps of $5 \mathrm{~nm}$ were used. As a rule of thumb the image with the focus in the middle of the sample corresponds to the projected potential along the beam waist at that depth (see Fig.1 middle image). The side view of the sample was extracted by stacking TFS images on top of each other and compared with a full multi-slice simulation (see Fig. 1 right image).

Figure 2 displays a side view of a $40 \mathrm{~nm}$ thick Si [110] sample obtained from a TFS iDPC-STEM sequence with focus steps of $5 \mathrm{~nm}$, starting at the bottom of the sample and moving towards the top. Local beam damage was induced by parking the probe for several seconds at a single position. Both the cross section and the images at three different depths reveal stronger beam damage at the bottom of the Si crystal (see Fig. 2).

\section{References:}

[1] E.G.T. Bosch \& I. Lazić, Ultramicroscopy 156 (2015), p. 59.

[2] I. Lazić, E.G.T. Bosch and S. Lazar, Ultramicroscopy 160 (2016), p. 265.

[3] I. Lazić, E.G.T. Bosch, Advances in Imaging and Electron Physics 199 (2017), p. 75.

[4] E. Yücelen, I. Lazić, E.G.T. Bosch, Scientific Reports 8 (2018), p. 2676.

[5] G. Behan et al, Phil. Trans. R. Soc. A 367 (2009), p. 3825.

[6] G. Saito et al, Ultramicroscopy 175 (2017), p. 97.

[7] J.J. Einspahr, P.M. Voyles, Ultramicroscopy 106 (2006), p. 1041. 


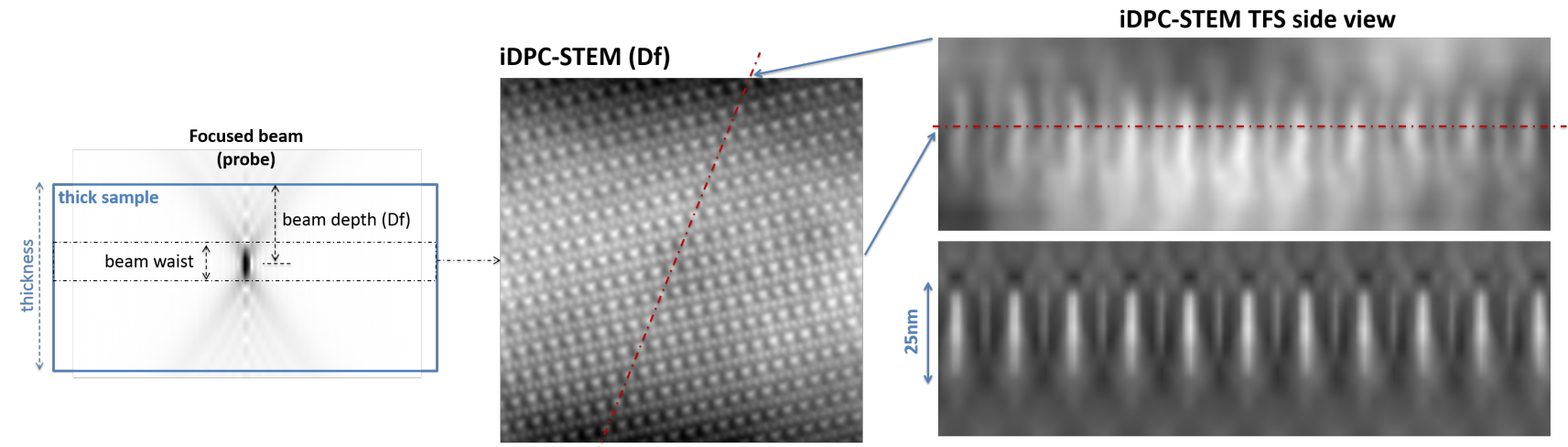

Figure 1. Schematic (left) and iDPC-STEM image (middle) of $25 \mathrm{~nm}$ thick $\mathrm{SrTiO}_{3}$ [011] sample. The beam cross-over was placed inside the sample approximately $10 \mathrm{~nm}$ from top surface. (right) Side view of TFS iDPC-STEM in steps of $5 \mathrm{~nm}$ of $\mathrm{SrTiO}_{3}$ [011]. Atomic columns, including oxygen, are clearly visible along the whole sample thickness. Top: experiment, Titan Themis $300 \mathrm{keV}$, probe corrected with 25 mrad opening angle. Bottom: Multi-slice iDPC-STEM simulation under the same conditions.

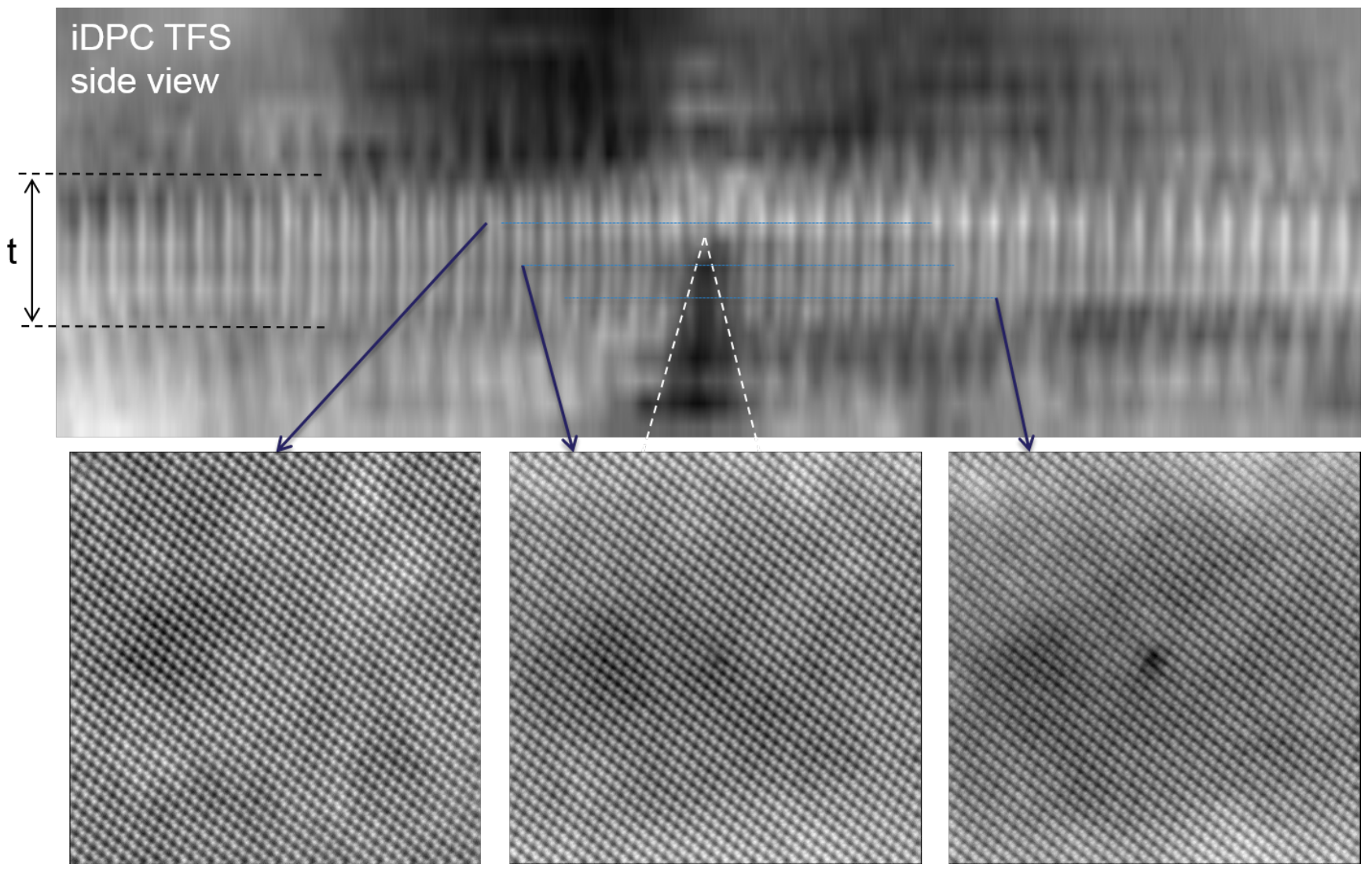

Figure 2. Top: Side view of a TFS iDPC-STEM stack of a $40 \mathrm{~nm}$ thick Si [110] sample. Images acquired on Titan Themis at $300 \mathrm{kV}$, probe corrected with $30 \mathrm{mrad}$ opening angle, in $5 \mathrm{~nm}$ focus steps going from bottom to top. The beam damage after parking the beam for several seconds at the center of the image, focusing near the bottom of the sample is clearly visible. Bottom: Three Si [110] images at three different depths of the sample. 